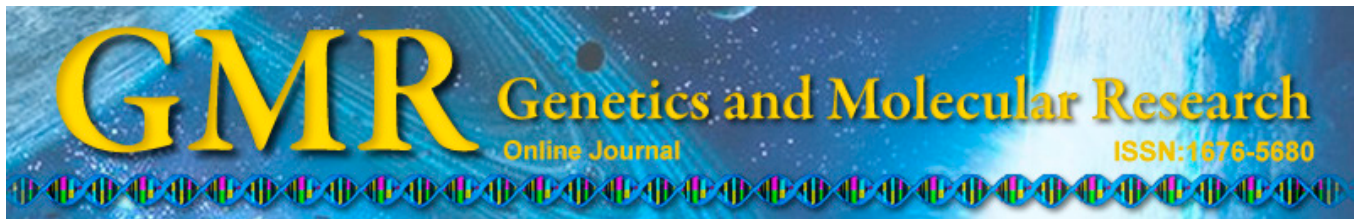

\title{
Proton pump inhibitor use and risk of spontaneous bacterial peritonitis in cirrhotic patients: a systematic review and meta-analysis
}

H.B. Xu ${ }^{1,3}$, H.D. Wang ${ }^{2}$, C.H. Li ${ }^{1}$, S. Ye ${ }^{1}$, M.S. Dong ${ }^{4}$, Q.J. Xia ${ }^{4}$, A.Q. Zhang ${ }^{1}$, K. Pan ${ }^{1}$, X.L. Ge ${ }^{1}$ and J.H. Dong ${ }^{1}$

${ }^{1}$ Hospital and Institute of Hepatobiliary Surgery, PLA General Hospital, Beijing, China

${ }^{2}$ Department of Hepatobiliary Surgery,

The Second Hospital of Dalian Medical University, Dalian, China

${ }^{3}$ Chinese PLA 211 Hospital, Harbin, China

${ }^{4}$ Department of General Surgery,

General Hospital of the PLA Second Artillery Force, Beijing, China

Corresponding author: J.H. Dong

E-mail: dongjh301@sina.com

Genet. Mol. Res. 14 (3): 7490-7501 (2015)

Received October 23, 2014

Accepted January 29, 2015

Published July 3, 2015

DOI http://dx.doi.org/10.4238/2015.July.3.25

ABSTRACT. We used a meta-analysis approach to investigate the association between proton pump inhibitor (PPI) use and risk of spontaneous bacterial peritonitis (SBP) in cirrhotic patients. We searched Ovid Medline, Embase, and the Cochrane Library to identify eligible studies. We included studies that compared cirrhotic patients who did or did not use PPIs. The primary outcome was SBP, and the secondary outcome was overall bacterial infection. Results were pooled using random-effect models. This process led to identification of 12 journal articles and 5 conference abstracts. The pooled data showed that PPI use in patients with cirrhosis and ascites was significantly associated with an increased risk of SBP [odds ratio $(\mathrm{OR})=2.17 ; 95 \%$ 
confidence interval $\left.(\mathrm{CI})=1.46-3.23 ; \mathrm{P}<0.05 ; \mathrm{I}^{2}=85.6 \%\right]$ and overall risk of bacterial infection $(\mathrm{OR}=1.98 ; 95 \% \mathrm{CI}=1.36-2.87 ; \mathrm{P}<0.05$; $\mathrm{I}^{2}=0$ ). Subgroup analysis revealed that journal articles and studies reporting adjusted effect estimates demonstrated that PPI users had a significantly increased risk of SBP $(\mathrm{OR}=2.13 ; 95 \% \mathrm{CI}=1.61-2.82 ; \mathrm{P}$ $<0.05 ; \mathrm{I}^{2}=29.4 \%$; and $\mathrm{OR}=1.98 ; 95 \% \mathrm{CI}=1.42-2.77 ; \mathrm{P}<0.05 ; \mathrm{I}^{2}=$ $67 \%$, respectively). In conclusion, PPI use increased the risk of SBP and overall bacterial infection in patients with cirrhosis and ascites. PPIs should be administered after careful assessment of the indications in cirrhotic patients. Future well-designed prospective studies are warranted to clarify the dose relationships and to compare infection risks associated with different classes of PPIs.

Key words: Cirrhosis; Meta-analysis; Proton pump inhibitor; Spontaneous bacterial peritonitis

\section{INTRODUCTION}

Since proton pump inhibitors (PPIs) were first introduced into clinical practice in the late 1980s, they have become well established as first-line drugs for the treatment of various gastric acid secretion disorders (Leontiadis et al., 2006). Notably, PPIs are prescribed widely for a large proportion of cirrhotic patients with various indications including peptic ulcers, gastroesophageal reflux disease, portal hypertensive gastropathy, gastrointestinal bleeding, and Barrett esophagus. In the early years, PPIs were perceived as safe medications. However, a growing number of recent studies have raised concerns about the increased risk of infection-related events that accompany PPI administration; these events include hospital-acquired pneumonia, Clostridium difficile infection, and spontaneous bacterial peritonitis (SBP) (Bajaj et al., 2009; Herzig et al., 2009; Linsky et al., 2010).

SBP is a very common, life-threatening bacterial infection in patients with cirrhosis and ascites (European Association for the Study of the Liver, 2010). Small intestinal bacterial overgrowth (SIBO) and translocation of bacteria from the intestinal lumen to the lymph nodes may contribute to SBP development (Chang et al., 1998; Ginès et al., 2004). Since PPIs are gastric acid secretion-suppressive drugs, they may facilitate the proliferation of intestinal bacteria (de Vos et al., 2013). PPIs may also impair gastrointestinal motility, which could predispose patients to SIBO and bacterial translocation across the intestinal epithelial barrier (van Vlerken et al., 2012; de Vos et al., 2013; Jacobs et al., 2013). Numerous studies have suggested that PPI use by cirrhotic patients may increase the risk of SBP (Bajaj et al., 2009; de Vos et al., 2013; Min et al., 2014; Ratelle et al., 2014). However, some studies have drawn opposite conclusions (Campbell et al., 2008; Aditi et al., 2012; Terg et al., 2012; van Vlerken et al., 2012).

Two previous meta-analyses have been carried out to address this controversial issue (Trikudanathan et al., 2011; Deshpande et al., 2013). However, both of these previous analyses were limited by their inclusion of a small number of studies and a lack of sufficient exploration of heterogeneity and bias. As a growing number of new studies have been published, we decided to perform an updated meta-analysis in order to investigate the association between PPI use and SBP risk in patients with cirrhosis and ascites. 


\section{MATERIAL AND METHODS}

\section{Search strategy}

The overview of this meta-analysis was conducted in accordance with the Preferred Reporting Items for Systematic Reviews and Meta-analysis statement (Moher et al., 2009). We performed an electronic search for all eligible studies reported from 1988 to August 2014 using Medline (Ovid), Embase, and the Cochrane Library. The following search terms were used: "anti-ulcer agent", "antacid", "proton pump inhibitor", "proton pumps", "PPI", "omeprazole", "rabeprazole", "lansoprazole", "pantoprazole", "esomeprazole", "peritonitis", "spontaneous bacterial peritonitis", "SBP", "cirrhosis", and "cirrhotic". The language was limited to English. We limited the search to studies reported after 1988, because that was the year PPIs were first introduced. We also manually searched major international gastroenterology conference abstracts and the references of selected articles to identify potentially relevant studies.

\section{Inclusion criteria}

The inclusion criteria for our meta-analysis were as follows: 1) observational studies, including cohort and case-control studies; 2) studies that investigated the association between PPI use and risk of SBP in cirrhotic patients that included more than 20 participants; 3) PPIs prescribed prior to occurrence of SBP; 4) SBP risk was evaluated as an endpoint. The authors had to report effect estimates [risk ratio (RR), hazard ratio (HR), or odds ratio (OR)] and 95\% confidence intervals (CIs) for the comparison of SBP risk between PPI users and PPI nonusers. If effect estimates were reported for more than one set of adjustments, we selected the most adjusted estimate. We also included studies with raw data reported in the comparison arms that could be used to calculate unadjusted effect estimates.

\section{Data collection and quality assessment}

Two independent reviewers (H.B. Xu and H.D. Wang) screened all titles and abstracts for eligibility, and all studies on PPI prescription in patients with cirrhosis were then retrieved. Studies were then independently assessed based on our predefined selection criteria. Disagreements between the two reviewers were resolved by discussion or by consultation with the corresponding author (J.H. Dong). The extracted data included the following information: first author's name, year of report, publication type, study design, location, sample size, mean patient age, inclusion criteria, exclusion criteria, definition of PPI use, percentage of inappropriate PPI indications, endpoint, adjustments, study period, and quality assessment score. The quality of cohort or case-control studies was evaluated using the Newcastle-Ottawa scale, as recommended by the Cochrane Non-Randomized Studies Methods (Wells et al., 2009).

\section{Statistical analysis}

All meta-analyses were performed using the STATA software (version 12.0; Stata Corporation, College Station, TX, USA). The ORs and their associated 95\%CIs were considered to be the effect sizes for all studies. Considering the low incidence of SBP events in PPI users, we assumed that HRs and RRs were similar to ORs (Davies et al., 1998). We 
preferred adjusted ORs for our analysis, since they account for confounding variables. If only raw data were available, we calculated the unadjusted OR accordingly. The DerSimonianLaird random-effects model was used to minimize the potential heterogeneity among studies. Heterogeneity among studies was mainly assessed using the $\mathrm{I}^{2}$ statistic. We considered low, moderate, and high $\mathrm{I}^{2}$ values to be 25,50 , and $75 \%$, respectively (Higgins et al., 2003). The potential source of heterogeneity was further explored by sensitivity and subgroup analyses. Meta-regression analysis was conducted using a random-effect model in order to investigate whether a specific covariate influenced the effect. The statistically significant covariates were then included in a multiple meta-regression. For cases of false-positive results, permutation tests were performed to calculate adjusted $\mathrm{P}$ values. We assessed publication bias quantitatively using the Egger test and qualitatively by visual inspection of funnel plots (Egger et al., 1997). A threshold of $\mathrm{P}<0.1$ was used to decide whether heterogeneity or publication bias existed. For other tests, $\mathrm{P}<0.05$ was considered to be statistically significant.

\section{RESULTS}

The process for selection of eligible studies is shown in Figure 1. The initial search yielded 233 citations, from which we selected 70 records as potential candidates after screening of titles and abstracts. We excluded 41 records because they did not report detailed data concerning PPI use. We also rejected 9 studies without sufficient SBP data to calculate the effect estimates and 2 conference posters that overlapped with journal articles. Therefore, 18 potentially relevant records were selected. One conference poster that reported 16 patients was excluded because of the small sample size. Finally, 17 studies were pooled for meta-analysis.

The included observational studies were published between 2008 and 2014 and involved 8204 patients. Twelve studies were published in journals (Campbell et al., 2008; Bajaj et al., 2009; Choi et al., 2011; Goel et al., 2012; van Vlerken et al., 2012; de Vos et al., 2013; Kwon et al., 2014; Merli et al., 2014; Min et al., 2014; Miura et al., 2014; O’Leary et al., 2014; Ratelle et al., 2014) and 5 studies were reported as conference abstracts (Northup et al., 2008; Bulsiewicz et al., 2009; Aditi et al., 2012; Terg et al., 2012; Mandorfer et al., 2014). We included 8 retrospective cohort studies, 6 case-control studies, and 3 prospective cohort studies. There were 8 studies conducted in North American populations, 4 in Asian populations, 4 in European populations, and 1 in a South American population. The predefined exclusion criteria varied across the studies, with gastrointestinal bleeding, prior antibiotic use, immunosuppressive conditions, and organ transplantation listed most frequently. Adjusted effect estimates were reported in 12 studies. However, the degree of adjustment differed greatly between studies. Commonly reported adjustments included age, gender, Model for End-Stage Liver Disease (MELD) score, Child-Pugh score (CPS), and serum sodium level. An unadjusted OR was reported in 1 poster (Northup et al., 2008) and was calculated from raw data in 4 studies. Overprescription of PPIs was reported in 10 studies, with inappropriate indication rates ranging from 19.7 to $86 \%$. The characteristics of the included studies are summarized in Table 1.

Cohort and case-control studies with available full-text were evaluated by the Newcastle-Ottawa scale. Most items had full agreement. Notably, most studies lost points in the item of comparability, as some baseline characteristics of clinical importance were not well balanced between comparison groups (Table 1). 


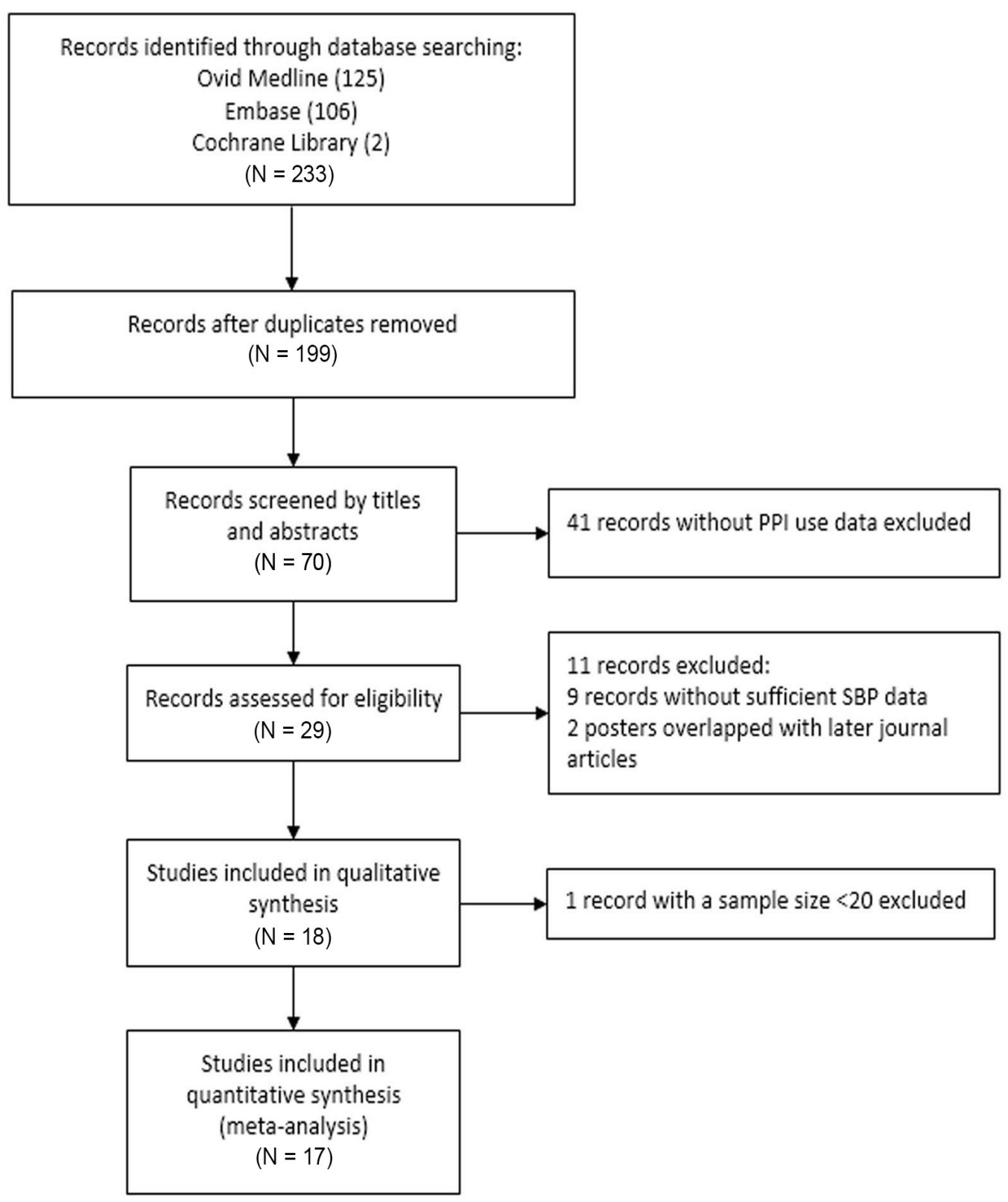

Figure 1. Selection process of eligible studies. 
Proton pump inhibitors in cirrhotic patients

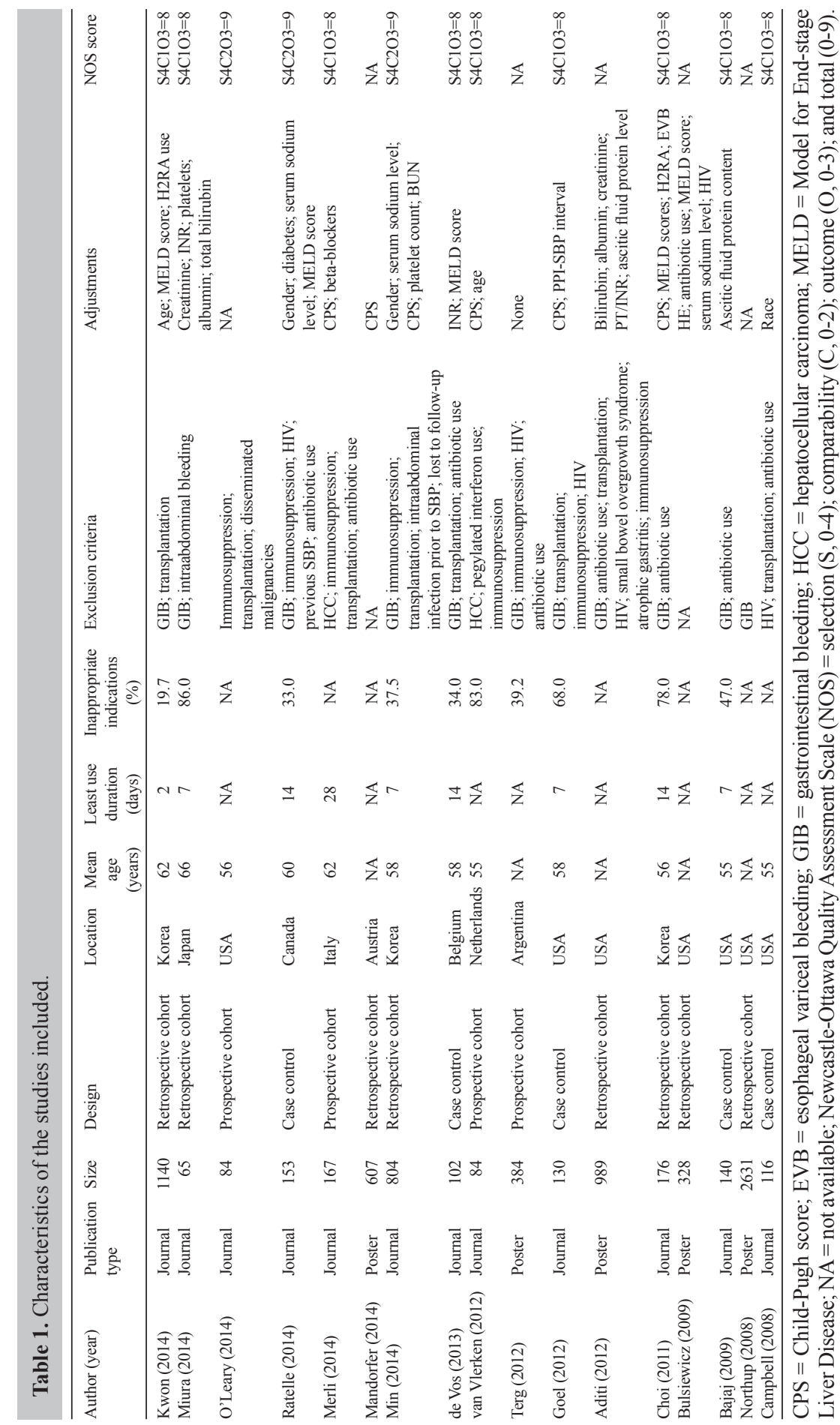




\section{PPI use and risk of SBP}

The pooled data showed a significant association between PPI use and risk of SBP in patients with cirrhosis and ascites $(\mathrm{OR}=2.17 ; 95 \% \mathrm{CI}=1.46-3.23 ; \mathrm{P}<0.05)$ accompanied by high heterogeneity $\left(\mathrm{I}^{2}=85.6 \%\right)$, as shown in Figure 2 . The funnel plot was symmetrical, suggesting a low likelihood of publication bias. The Egger test also did not indicate the presence of publication bias $(\mathrm{P}=0.37)$.

The high heterogeneity was explored by sensitivity analysis, stratification analyses, and meta-regression analyses. In the sensitivity analysis, removing the study by Northup et al. (2008) altered the summary statistic to the greatest extent, whereas the overall significance did not materially change $\left(\mathrm{OR}=1.89 ; 95 \% \mathrm{CI}=1.39-2.58 ; \mathrm{P}<0.05 ; \mathrm{I}^{2}=67.2 \%\right)$. Subgroups were stratified according to publication type, study design, population, and adjustment of effect estimates. Notably, both journal articles and studies that reported adjusted effect estimates indicated that PPI users had a significantly increased risk of SBP $(\mathrm{OR}=2.13 ; 95 \% \mathrm{CI}$ $=1.61-2.82 ; \mathrm{P}<0.05 ; \mathrm{I}^{2}=29.4 \% ;$ and $\mathrm{OR}=1.98 ; 95 \% \mathrm{CI}=1.42-2.77 ; \mathrm{P}<0.05 ; \mathrm{I}^{2}=67 \%$, respectively). However, subgroup analyses of conference abstracts, prospective cohorts, the South American population, and unadjusted ORs were not statistically significant (Table 2). The publication year and sample size were considered to be independent variables in the metaregression analysis. Neither sample size nor publication year had an independent effect on the SBP outcome $(\mathrm{P}=0.53$ and $\mathrm{P}=0.22$, respectively).

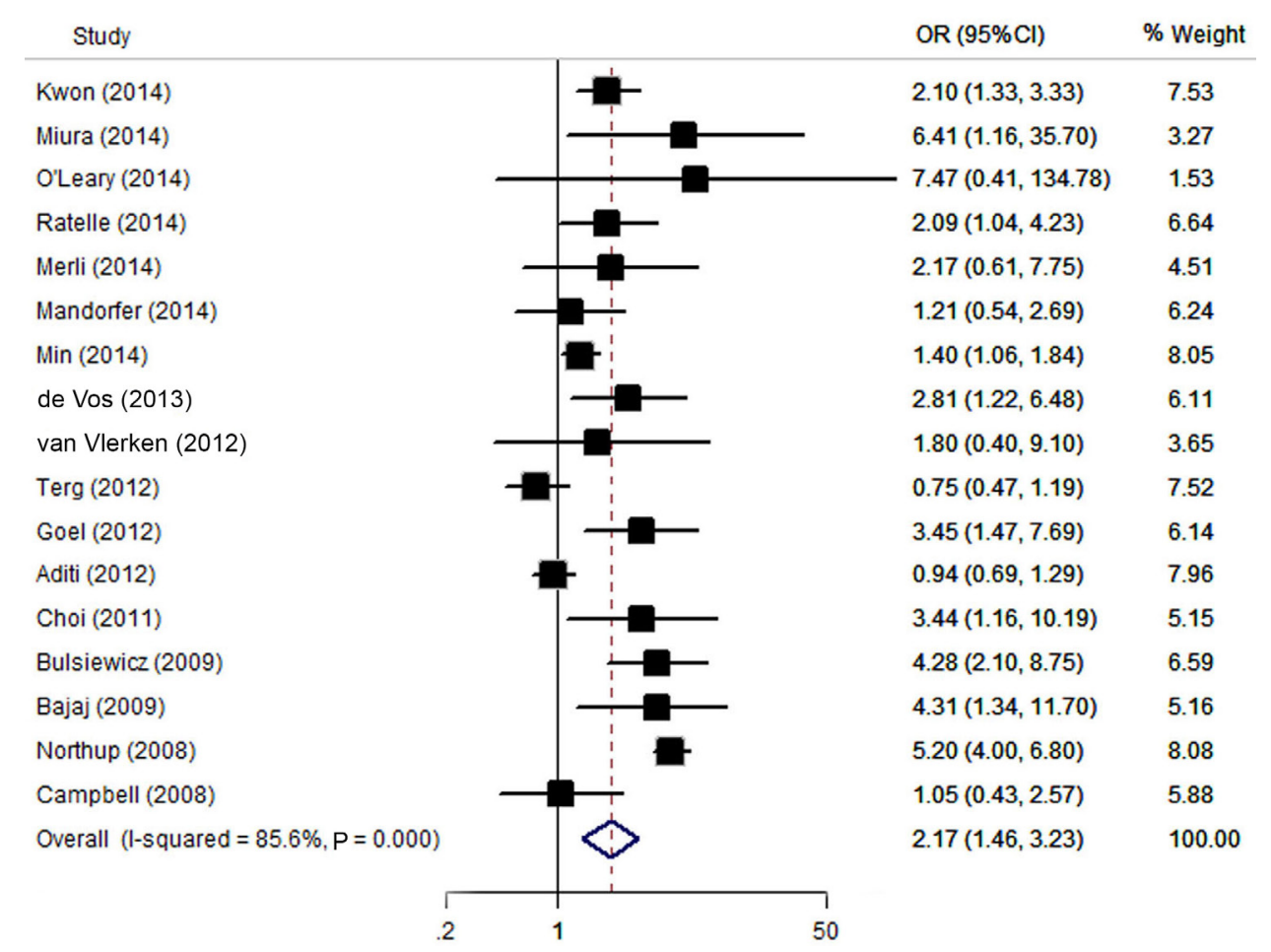

Figure 2. Forest plot illustrating the association between proton pump inhibitor use and risk of spontaneous bacterial peritonitis in cirrhosis. 
Table 2. Subgroup analyses for studies investigating the risk of SBP in PPI users with cirrhosis.

\begin{tabular}{|c|c|c|c|c|c|}
\hline Subgroups & No. of studies & OR & $95 \%$ CI & $P$ value & $\mathrm{I}^{2}(\%)$ \\
\hline \multicolumn{6}{|l|}{ Publication type } \\
\hline Journal & 12 & 2.13 & $1.61-2.82$ & $<0.001$ & 29.4 \\
\hline Conference abstracts & 5 & 1.80 & $0.71-4.58$ & 0.217 & 95.8 \\
\hline \multicolumn{6}{|l|}{ Study design } \\
\hline Retrospective cohort & 8 & 2.33 & $1.30-4.17$ & 0.004 & 91.9 \\
\hline Case control & 6 & 1.91 & $1.03-3.55$ & 0.040 & 74.7 \\
\hline Prospective cohort & 3 & 2.31 & $0.91-5.87$ & 0.079 & 0 \\
\hline \multicolumn{6}{|l|}{ Populations } \\
\hline Asian & 4 & 2.01 & $1.26-3.21$ & 0.003 & 54.5 \\
\hline North American & 8 & 2.64 & $1.31-5.30$ & 0.007 & 90.6 \\
\hline European & 4 & 1.86 & $1.13-3.07$ & 0.015 & 0 \\
\hline South American & 1 & 0.75 & $0.47-1.19$ & 0.225 & - \\
\hline \multicolumn{6}{|l|}{ Adjustment } \\
\hline Adjusted & 12 & 1.98 & $1.42-2.77$ & $<0.001$ & 67 \\
\hline Unadjusted & 5 & 2.47 & $0.84-7.31$ & 0.102 & 92.2 \\
\hline
\end{tabular}

Significant $\mathrm{P}$ values were shown in bold.

\section{PPI use and overall risk of infection}

There were 3 studies that also evaluated the association between PPI use and overall risk of bacterial infection in cirrhotic patients (van Vlerken et al., 2012; Merli et al., 2014; O'Leary et al., 2014). The aggregated data showed that PPI use was significantly associated with a higher overall risk of bacterial infection $(\mathrm{OR}=1.98 ; 95 \% \mathrm{CI}=1.36-2.87 ; \mathrm{P}<0.05$; Figure 3). There was no evidence of heterogeneity $\left(\mathrm{I}^{2}=0\right)$.

\begin{tabular}{|c|c|c|}
\hline Study & OR $(95 \% \mathrm{Cl})$ & $\%$ Weight \\
\hline O'Leary (2014) & $2.72(1.30,5.71)$ & 25.26 \\
\hline Merli (2014) & $2.00(1.20,3.20)$ & 57.51 \\
\hline van Vlerken (2012) & $1.20(0.50,3.00)$ & 17.23 \\
\hline Overall $(\mathrm{I}$-squared $=0.0 \%, \mathrm{P}=0.385$ ) & $1.98(1.36,2.87)$ & 100.00 \\
\hline $\begin{array}{l}5 \\
.2\end{array}$ & $\sqrt{7}$ & \\
\hline
\end{tabular}

Figure 3. Forest plot illustrating the association between proton pump inhibitor use and risk of overall infections in cirrhosis.

\section{Dose and duration of PPI use}

Min et al. (2014) reported that a standard dose of PPI increased the risk of SBP more than a half dose $(\mathrm{HR}=2.184 ; 95 \% \mathrm{CI}=0.935-5.103 ; \mathrm{P}=0.07)$. However, another study that evaluated the overall bacterial infection risk found no difference in the PPI doses administered to patients with or without infections (van Vlerken et al., 2012). Dose effects could not be 
quantitatively analyzed because data were insufficient and inconsistent dosages were used across the different studies. There were 9 studies (59\%) that defined the shortest duration of PPI use, ranging from 2 days to 4 weeks (Table 1). We could not perform a stratified analysis of duration because of inconsistent definitions. Notably, Choi et al. (2011) compared patients who used PPIs for the median duration of time to PPI non-users, deducing that longer duration of PPI use may be associated with an increased risk of SBP. Goel et al. (2012) found that patients who had taken PPIs 8 to 90 days before hospitalization for cirrhosis were $79 \%$ less likely to develop SBP than those who had taken PPIs within 7 days. Later, Kwon et al. (2014) showed that both PPI use 8 to 30 days before hospitalization for cirrhosis as well as PPI use within 7 days before hospitalization significantly increased the risk of SBP $(\mathrm{P}=0.009$ and $\mathrm{P}$ $=0.001$, respectively).

\section{DISCUSSION}

Our meta-analysis was based on 17 observational studies that included over 8000 patients with cirrhosis and ascites. The pooled data showed that PPI use was associated with an approximately 2-fold increased risk of developing SBP for cirrhotic patients. We analyzed additional data provided by 3 studies and found that PPI use was also associated with an increased overall risk of bacterial infection.

The high heterogeneity among studies was explored by subgroup analyses and metaregression analyses. Notably, the effect remained pronounced in the subgroup of journal articles, but not in the subgroup of conference abstracts. Since conference posters likely include more heterogeneous characteristics and could not be assessed for methodological quality, they may serve as a potential source of heterogeneity. When we stratified the studies based on adjustments, only studies with adjusted ORs yielded significant results. This discrepancy implies that uncontrolled potential confounding variables may contribute to the observed heterogeneity. Although the subgroup of prospective studies yielded no statistically significant results, this should be interpreted with caution because 2 of the studies reported unadjusted effect estimates. Our meta-regression analysis found that neither publication year nor sample size significantly affected outcomes.

PPI use in patients without acceptable indications was common across the included studies, ranging from 19.7 to $86 \%$ of patients. PPIs have been perceived as well-tolerated and safe drugs for quite some time, which has led to widespread overprescription. In cirrhotic patients, the main reason for inadequate PPI use was previous variceal bleeding (Kalaitzakis and Björnsson, 2008). Although clinical guidelines recommended PPI administration prior to endoscopic variceal ligation, this is not reasonable for continuous long-term PPI therapy (Garcia-Tsao et al., 2007; Siple et al., 2012). Acid secretion is constitutively reduced during cirrhosis, so there is no sufficient evidence to support PPI use for prophylaxis of peptic complications in patients with portal hypertension or esophageal varices (Merli et al., 2014). Additionally, nonspecific symptoms such as abdominal pain and dyspepsia were common inadequate indications for PPI use (Kalaitzakis and Björnsson, 2008).

The mechanism that explains the correlation between PPI use and SBP remains unclear. Several lines of investigation have hypothesized that PPIs could increase SIBO. In combination with the impaired intestinal permeability and motility of cirrhotic patients, SIBO may lead to bacterial translocation and subsequent SBP (Bajaj et al., 2009; Jacobs et al., 2013). However, conflicting results have been reported concerning the association between PPI use, 
intestinal permeability, and SIBO (Ratuapli et al., 2012; van Vlerken et al., 2012). One study that compared patients who had taken PPIs in the previous 7 days to patients who had taken PPIs in the previous 8 to 90 days found that only patients who had taken PPIs in the previous 7 days were at risk of developing SBP (Goel et al., 2012). Since SIBO rarely develops in a shorter period, other mechanisms may be implicated. For instance, it has been suggested that PPIs may inhibit neutrophil function and natural killer cell activity (Zedtwitz-Liebenstein et al., 2002). Also, they may decrease the cellular oxidative burst (Garcia-Martinez et al., 2015).

We are aware of 2 previous meta-analyses that are relevant to this topic. The earliest meta-analysis, published in 2011, only included 4 studies that examined 772 patients (Trikudanathan et al., 2011). The more recent meta-analysis only included 8 studies (Deshpande et al., 2013). Both studies failed to explore sources of heterogeneity and did not evaluate data concerning dose or duration. Also, overall infection risk was not one of the analyzed outcomes. When designing our study, we sought to overcome these previous limitations. We incorporated all relevant studies that we could identify through August 2014, which added a series of newly published studies with a very large number of patients, thereby substantially increasing the power of our analysis. We also explored sources of heterogeneity by employing subgroup, sensitivity, and meta-regression analyses. The heterogeneous dose and duration results were systematically reviewed, and overall infection risk was evaluated.

Despite these strengths, our study does have some limitations that should be acknowledged. We only included studies written in English, so relevant studies published in non-English language journals may have been excluded. Also, studies with statistically significant results were more likely to be published and cited by English language publishers. For a more comprehensive approach, we included several conference abstracts. However, the quality of these abstracts could not be assessed, and it is possible that important study information was not considered. Moreover, only observational studies, most of which were retrospective studies, were available for our meta-analysis. Information concerning PPI use was primarily obtained from previous prescription records; therefore, some PPI users may have been excluded because of unclear medication records. Also, recall bias could lead to an underestimation of PPI users. It is not possible to establish causal relationships based on observational studies. The various studies included in our analysis did not use the same predefined PPI indications, inclusion criteria, and exclusion criteria. It is also important to note that cirrhotic patients who already have a higher risk of SBP may be taking PPIs. For example, cirrhotic patients with dyspepsia caused by impaired intestinal motility may have pre-existing SIBO, predisposing them to SBP (Bajaj et al., 2009). Not all studies excluded patients who had received prior antibiotic treatment and patients with immunosuppressive conditions (Bajaj et al., 2009; Choi et al., 2011; Goel et al., 2012). It is also notable that the included studies did not adjust for the same confounders, and they generally failed to account for one or more of the following SBP risk factors: increased age, low fluid protein concentration, high MELD score or CPS, and increased international normalized ratio. Additionally, residual confounding from unmeasured covariates could not be ignored, even after multivariate analysis. Furthermore, most studies reported limited data regarding PPI dose and duration, which precluded subgroup analyses based on these profiles and dose-response analysis. It is also possible that different types of PPIs function differently. Finally, this meta-analysis was performed at a study level. We were not able to address or incorporate individual factors at the patient level. 


\section{CONCLUSIONS}

Our meta-analysis of observational studies found that PPI use was associated with an approximately 2-fold increased risk of SBP in patients with cirrhosis and ascites. PPI use was also potentially associated with an increased overall risk of bacterial infection. Our results incorporated information from all updated studies with large sample sizes, which reinforced the power greatly. PPIs should be used judiciously with regard to appropriate indications and duration in cirrhotic patients. Future well-designed prospective studies are needed to clarify potential causal relationships between PPI use and SBP as well as the impact of dosage and duration.

\section{REFERENCES}

Aditi A, Crippin JS and Abhishek A (2012). Role of proton pump inhibitors in the development of spontaneous bacterial peritonitis amongst cirrhotics; a retrospective cohort study. Gastroenterology 142: S946.

Bajaj JS, Zadvornova Y, Heuman DM, Hafeezullah M, et al. (2009). Association of proton pump inhibitor therapy with spontaneous bacterial peritonitis in cirrhotic patients with ascites. Am. J. Gastroenterol. 104: 1130-1134.

Bulsiewicz WJ, Scherer JR, Feinglass JM, Howden CW, et al. (2009). Proton pump inhibitor (PPI) use is independently associated with spontaneous bacterial peritonitis (SBP) in cirrhotics with ascites. Gastroenterology 136: A11.

Campbell MS, Obstein K, Reddy KR and Yang YX (2008). Association between proton pump inhibitor use and spontaneous bacterial peritonitis. Dig. Dis. Sci. 53: 394-398.

Chang CS, Chen GH, Lien HC and Yeh HZ (1998). Small intestine dysmotility and bacterial overgrowth in cirrhotic patients with spontaneous bacterial peritonitis. Hepatology 28: 1187-1190.

Choi EJ, Lee HJ, Kim KO, Lee SH, et al. (2011). Association between acid suppressive therapy and spontaneous bacterial peritonitis in cirrhotic patients with ascites. Scand. J. Gastroenterol. 46: 616-620.

Davies HT, Crombie IK and Tavakoli M (1998). When can odds ratios mislead? BMJ 316: 989-991.

de Vos M, De Vroey B, Garcia BG, Roy C, et al. (2013). Role of proton pump inhibitors in the occurrence and the prognosis of spontaneous bacterial peritonitis in cirrhotic patients with ascites. Liver Int. 33: 1316-1323.

Deshpande A, Pasupuleti V, Thota P, Pant C, et al. (2013). Acid-suppressive therapy is associated with spontaneous bacterial peritonitis in cirrhotic patients: a meta-analysis. J. Gastroenterol. Hepatol. 28: 235-242.

Egger M, Davey Smith G, Schneider M and Minder C (1997). Bias in meta-analysis detected by a simple, graphical test. BMJ 315: 629-634.

European Association for the Study of the Liver (2010). EASL clinical practice guidelines on the management of ascites, spontaneous bacterial peritonitis, and hepatorenal syndrome in cirrhosis. J. Hepatol. 53: 397-417.

Garcia-Martinez I, Francés R, Zapater P, Giménez P, et al. (2015). Use of proton pump inhibitors decrease cellular oxidative burst in patients with decompensated cirrhosis. J. Gastroenterol. Hepatol. 30: 147-154.

Garcia-Tsao G, Sanyal AJ, Grace ND, Carey W, et al. (2007). Prevention and management of gastroesophageal varices and variceal hemorrhage in cirrhosis. Hepatology 46: 922-938.

Ginès P, Cárdenas A, Arroyo V and Rodés J (2004). Management of cirrhosis and ascites. N. Engl. J. Med. 350: 16461654.

Goel GA, Deshpande A, Lopez R, Hall GS, et al. (2012). Increased rate of spontaneous bacterial peritonitis among cirrhotic patients receiving pharmacologic acid suppression. Clin. Gastroenterol. Hepatol. 10: 422-427.

Herzig SJ, Howell MD, Ngo LH and Marcantonio ER (2009). Acid-suppressive medication use and the risk for hospitalacquired pneumonia. JAMA 301:2120-2128.

Higgins JP, Thompson SG, Deeks JJ and Altman DG (2003). Measuring inconsistency in meta-analyses. BMJ 327: 557-560.

Jacobs C, Coss Adame E, Attaluri A, Valestin J, et al. (2013). Dysmotility and proton pump inhibitor use are independent risk factors for small intestinal bacterial and/or fungal overgrowth. Aliment. Pharmacol. Ther. 37: 1103-1111.

Kalaitzakis E and Björnsson E (2008). Inadequate use of proton-pump inhibitors in patients with liver cirrhosis. Eur. J. Gastroenterol. Hepatol. 20: 512-518.

Kwon JH, Koh SJ, Kim W, Jung YJ, et al. (2014). Mortality associated with proton pump inhibitors in cirrhotic patients with spontaneous bacterial peritonitis. J. Gastroenterol. Hepatol. 29: 775-781.

Leontiadis GI, Sharma VK and Howden CW (2006). Proton pump inhibitor treatment for acute peptic ulcer bleeding. Cochrane Database Syst. Rev.: CD002094.

Linsky A, Gupta K, Lawler EV, Fonda JR, et al. (2010). Proton pump inhibitors and risk for recurrent Clostridium difficile 
infection. Arch. Intern. Med. 170: 772-778.

Mandorfer M, Bota S, Schwabl P, Bucsics T, et al. (2014). Proton pump inhibitor treatment is neither associated with spontaneous bacterial peritonitis development nor with mortality in cirrhotic patients with ascites. J. Hepatol. 60: S393.

Merli M, Lucidi C, Di Gregorio V, Giannelli V, et al. (2014). The chronic use of beta-blockers and proton pump inhibitors may affect the rate of bacterial infections in cirrhosis. Liver Int. 35: 362-369.

Min YW, Lim KS, Min BH, Gwak GY, et al. (2014). Proton pump inhibitor use significantly increases the risk of spontaneous bacterial peritonitis in 1965 patients with cirrhosis and ascites: a propensity score matched cohort study. Aliment. Pharmacol. Ther. 40: 695-704.

Miura K, Tanaka A, Yamamoto T, Adachi M, et al. (2014). Proton pump inhibitor use is associated with spontaneous bacterial peritonitis in patients with liver cirrhosis. Intern. Med. 53: 1037-1042.

Moher D, Liberati A, Tetzlaff J, Altman DG, et al. (2009). Preferred reporting items for systematic reviews and metaanalyses: the PRISMA statement. PLoS Med. 6: e1000097.

Northup PG, Argo CK and Berg CL (2008). Chronic proton pump inhibitor use is strongly associated with hepatorenal syndrome and spontaneous bacterial peritonitis in cirrhosis patients. Hepatology 48: S325A.

O'Leary JG, Reddy KR, Wong F, Kamath PS, et al. (2014). Long-term use of antibiotics and proton pump inhibitors predict development of infections in patients with cirrhosis. Clin. Gastroenterol. Hepatol. 13: 753-759

Ratelle M, Perreault S, Villeneuve JP and Tremblay L (2014). Association between proton pump inhibitor use and spontaneous bacterial peritonitis in cirrhotic patients with ascites. Can. J. Gastroenterol. Hepatol. 28: 330-334.

Ratuapli SK, Ellington TG, O’Neill MT, Umar SB, et al. (2012). Proton pump inhibitor therapy use does not predispose to small intestinal bacterial overgrowth. Am. J. Gastroenterol. 107: 730-735.

Siple JF, Morey JM, Gutman TE, Weinberg KL, et al. (2012). Proton pump inhibitor use and association with spontaneous bacterial peritonitis in patients with cirrhosis and ascites. Ann. Pharmacother. 46: 1413-1418.

Terg R, Casciato PC, Garbe C, Cartier M, et al. (2012). Proton pump inhibitor therapy does not increase the incidence of bacterial infection in decompensated cirrhotic patients. A nationwide multicenter, prospective study. Hepatology 56: 255A.

Trikudanathan G, Israel J, Cappa J and O'Sullivan DM (2011). Association between proton pump inhibitors and spontaneous bacterial peritonitis in cirrhotic patients - a systematic review and meta-analysis. Int. J. Clin. Pract. 65: 674-678.

van Vlerken LG, Huisman EJ, van Hoek B, Renooij W, et al. (2012). Bacterial infections in cirrhosis: role of proton pump inhibitors and intestinal permeability. Eur. J. Clin. Invest. 42: 760-767.

Wells G, Shea B, O'Connell D, Peterson J, et al. (2009). The Newcastle-Ottawa Scale (NOS) for assessing the quality of nonrandomised studies in meta-analyses. http://www.ohri.ca/programs/clinical_epidemiology/oxford.asp. Accessed August 2014.

Zedtwitz-Liebenstein K, Wenisch C, Patruta S, Parschalk B, et al. (2002). Omeprazole treatment diminishes intra- and extracellular neutrophil reactive oxygen production and bactericidal activity. Crit. Care Med. 30: 1118-1122. 